\title{
Digital Media Design Strategy for Stage Based on Four Orders of Design*
}

\author{
Xiaoxi Liu ${ }^{1 * *}$, YansongChen ${ }^{2}$ \\ ${ }^{1}$ School of Film and Cinematic Arts, Faculty of Arts, Communication University of China, Beijing, 100024, \\ China \\ ${ }^{2}$ Academy of Arts and Design, Tsinghua University, Beijing, 100084, China \\ "Supported by "the Fundamental Resaerch Funds for the Central Universities"
}

\begin{abstract}
In the new media age, digital media technologies, manifested in LED screen, digital projection, artistic installation, digital lighting and other forms have gradually become the new trend in the development of stage design, which is continuously enriching the artistic expressions of stage performances. But new difficulties in stage design arises as digital media technologies become diversified and various professional fields on the stage design become integrated. Four orders of design is a fundamental theory to guide practice. Its integrated design ideas for building systematicity are of vital importance to direct the application of digital media in stage design. By analyzing the core content of four orders of design and reviewing the development tendency of digital media in stage design, this thesis seeks to delve into the role and connotation of the four orders of design in different aspects of digital media in stage design, and ultimately presents methods and strategies for applying digital media in stage design.
\end{abstract}

\section{The theory of "Four Orders of Design"}

Richard Buchanan has described the changing places of design practice and thinking as moving through four orders of design theory. ${ }^{1}$ He presents that the first order of design is visual communication with "symbols". The second order of design is the design of objects as "things". The third order of design he calls it "actions" that design activities and processes. The fourth order of design "thoughts" is the design of the environments and systems within which all the other orders of design coexist. ${ }^{2}$

Nowadays, profound background knowledge as well as in-depth thinking and analysis are required more in comprehensive design work in the field of architecture design, stage design, game design and so forth. The abovementioned four orders of design proposed by Richard Buchanan provides designers in different professional fields with an ever-deepening design idea compatible with development.

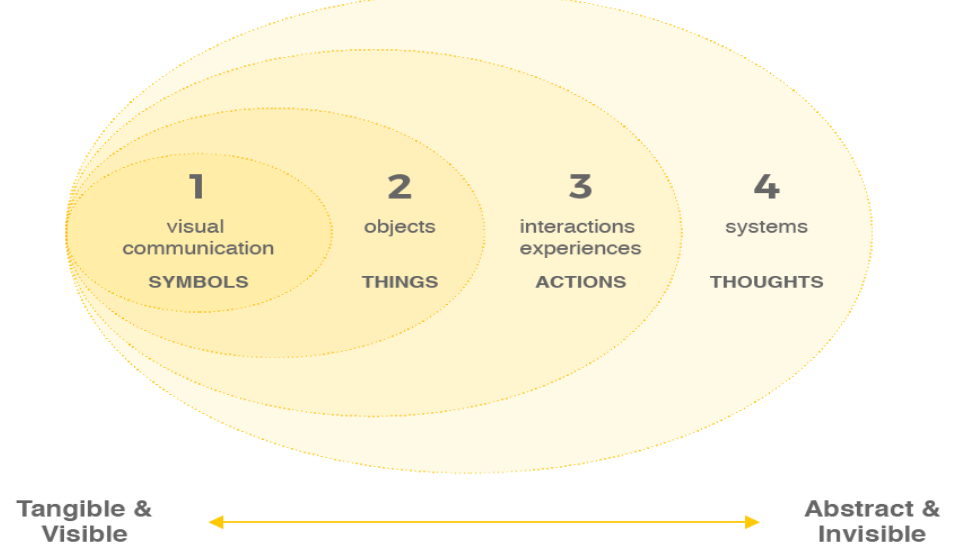

Figure 1. Four Orders of Design

\footnotetext{
** Corresponding author's e-mail:1xxcici@163.com
} 
Four orders of design are a process from tangible to abstract, like digital media design which focuses on systemic integration. Therefore, digital media designers for stage need to have the ability to deal with these very complex questions that lie at the core of four orders of design.

\section{The development of stage art design}

Stage serves as a space for performers and a focal point for audience. The form and content of stage has been constantly changing with time. The early stage dates back to the ancient Greek and Roman periods. Priene Theatre, built in 4th century B.C., is a classic model of ancient Greek theater. The design of its central stage is rather simple without any stage setting or advanced audio equipment. In the Renaissance, architects, painters, sculptors, more and more professionals from different fields, began to engage in stage design. The once simple stage was gradually transformed into more complicated structure as presented in proscenium stage, enhancing stereoscopic effect. Until around the World War II, the black box theater emerged in stage design field, which made stage becomes more diversified. Nowadays, in the context of new media age, stage design is constantly inspired by new materials and new technologies. Among which, the application of digital media technologies in stage art provides new idea for contemporary stage design. In the meantime, it transforms the thinking pattern and presentation of stage design from traditional static setting to dynamic and multi-dimensional expression.

\subsection{Traditional stage design}

Stage design is a time-and-space complex consisting of elements like space, shape, lighting, sense and activity etc. ${ }^{3}$ Traditional stage design can be divided into six parts: scene, construction, lighting, sound, costume and makeup. ${ }^{4}$ Therein, scene, costume and lighting is the foundation and core of traditional stage design. ${ }^{5}$ Scene is the major element to create space on stage as well as the major content to form external visual image. Lighting, on the one hand, is to illuminate. One the other hand, as the soul of stage design, lighting serves the plot to reshape the stage environment, render the space atmosphere and express the emotions of the characters. Costume is one of the major approaches to mould actors. Since costume directly demonstrates the external image of the characters, it plays a key role in traditional stage design. Construction is a great support for performance as actor could present certain environment and plot with the help of construction on stage. Stage make-up is an artistic creation based on the personality of the characters and the aesthetic imagination towards the characters. Sound, a non-verbal symbolic listening experience, together with visual effect, promotes audiences' better understanding towards the narration of the play.

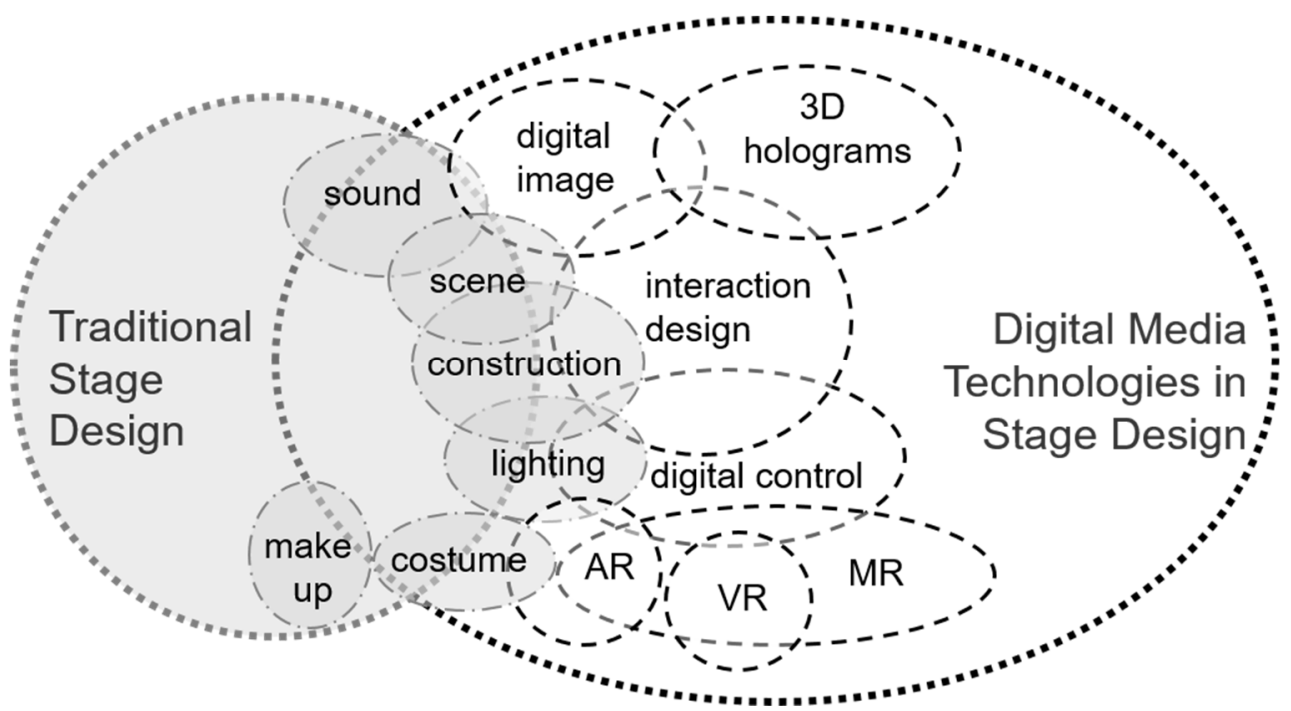

Figure 2. Traditional stage design and digital media technologies in stage design

\subsection{Development and application of digital media technologies in stage design}

Arnold Aronson, a professor from Columbia University, once proposed a concept "The Dematerialization of the Stage", which means adopting new media approach to dispel the material existence of the stage itself. ${ }^{6}$ Therefore, digital media technologies have gradually become significant means to dematerialize the stage and innovate stage art.
Among all the technologies in digital media, digital image has already become the most regular approach to create scene and artistic ambience in contemporary stage art. By employing pre-recorded digital image and various forms of digital media, designers could adjust different scenes on the stage in an easier and faster way. Functioning as the extension of stage experience space and regeneration of virtual space on stage, technologies like virtual reality(VR), augmented reality (AR) and mixed reality (MR) have gradually been applied to stage art design, and will become the significant method to upgrade stage art to a higher level to improve audiences' experience. Interaction technologies can create multi-tier 
connection among stage scenes, performers and audiences, thus establishing interaction with audiences not only in form but also in emotion level. Nowadays, strictly speaking, 3D holograms used on stage do not contain real three-dimensional image information. Rather, it is an illusion technique used already on stage in 1860 known as Pepper's Ghost, which projects a two-dimensional image disguised in three-dimensional shape on a transparent flat medium, thereby forming a three-dimensional visual effect on stage. With the advancement of technology, the $3 \mathrm{D}$ holograms presented on the stage now have more choices in the implementation methods than in the years when the Pepper's Ghost was used, and have achieved a qualitative improvement in achieving the effect. In 2016, on the stage of America's Got Talent, it is the 3D holograms that Sila Sveta used to present his multimedia performance "Pygmalion" (figure3). This technique reinforces the expression of stage, and renders spatial interaction and connection between illusion and performer. Powered by digital control technology, stage art installation can not only be the construction, but also act as a part of the scene. Besides, it realizes various machinery shifts, image changes, lighting and sound switches, and gradually becomes an important component in stage design.

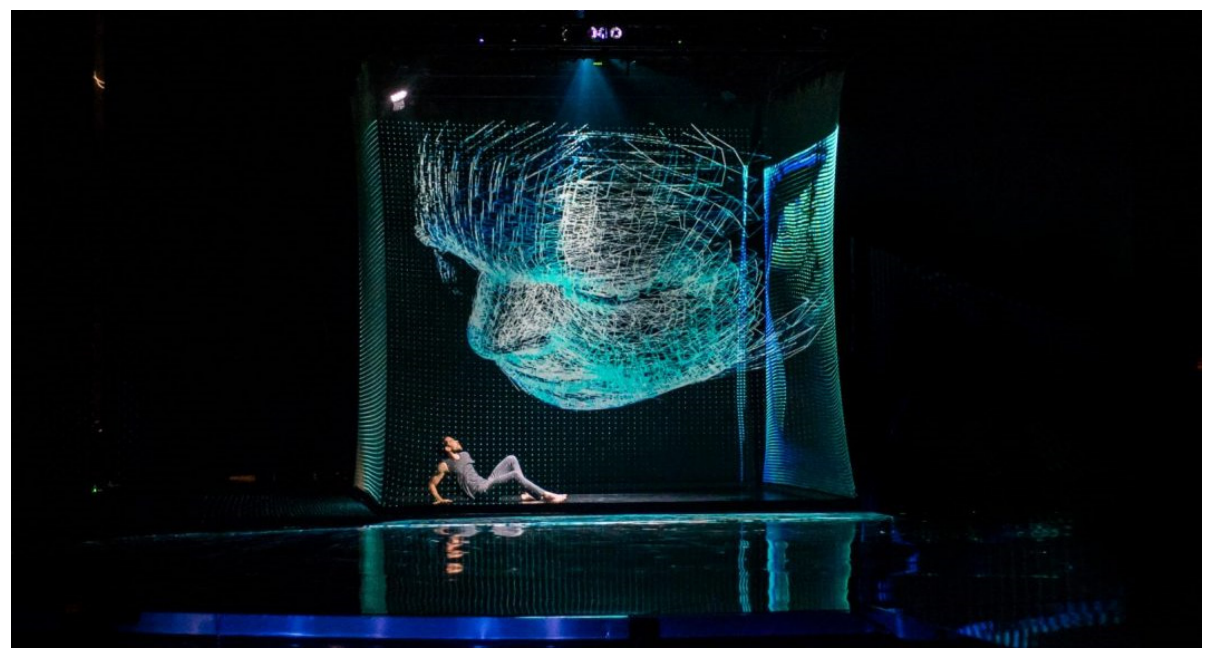

Figure 3. 3D holograms effect of multimedia performance "Pygmalion" stage

\section{Development of digital media design for stage under the four orders of design}

From building simple surroundings to dematerialization of the stage, the development of stage design is also manifested in the continuous process of digital media technologies applying to stage deign. This process is similar to four orders of design in terms of its tangible to abstract progressive procedure, and also in line with the overall design idea of establishing a connective design and developing systematic relationships in the design process.

\subsection{Conversion: partial focus to overall consideration}

Traditional stage design assignments have six relatively clear divisions: scene, construction, lighting, sound, costume, and make-up. However, in the backdrop of new media age, the role of digital media technologies in stage design is more like a pivot. Especially with the wide application of LED screen, LED lighting and projection, the boundaries between scene, construction, lighting, sound become vaguer, completing an integration of visual and auditory elements, scene atmosphere and interaction between actors and environments. Even in some scenes, through the motion capture and facial recognition of actors, the projection technologies also directly participate in shaping characters, realizing the conversion of the partial stage design to the overall stage design, and meanwhile provide more possibility for stage design.

\subsection{Development: from specialization to interdisciplinarity}

Digital media design itself is a comprehensive art form that integrates science and technology and artistic aesthetics. With its interdisciplinary characteristics, its application in stage design also promotes continuous innovation in stage art with regard to form and content. In traditional stage design, designers would, in line with performance content requirement, carefully paint the scene, design the lighting, create stage sound and make various props and costumes, thus realizing the final effect the stage requires. However, with wide application of digital technologies on stage, an increasingly diversified and comprehensive design system gradually achieves the deep cross-over among different departments of the stage. For example, the integration of multichannel digital audio control and space scene on stage creates a spatial stereo field in a better way for the integration could change digital lighting effect according to the change of music rhythm to create a more favorable audiovisual experience; the digital image corresponding with the space scenes and actor's movement could create an alternation between reality and illusion. 


\subsection{Transformation: from pre-production to real- time interaction}

Stage art has never been static, since static visual image and fixed scene clearly could not meet the need of contemporary stage art. In traditional stage design practice, designers from different departments would make an accurate plan and design for scene, construction, lighting, sound, costumes and make-up in advance within certain time and space. However, with the ongoing development of digital media technologies, interactive technologies based on radar positioning, infrared tracking, motion capture, audio frequency changes, light sensor, and mechanical control are gradually being applied to the stage, which establish a correlative system connecting certain action and space scene so that scenes, lights, sounds and other content can be changed according to the real-time data set in the stage interaction system to create natural dynamic scenes enhancing the artistic expression of the stage.

\subsection{Evolution: from atmosphere creation to narrative expression}

Owing to technical limitations, scene, construction, lighting, sound, costume, make-up in traditional stage design only carry relatively restricted messages, and their main roles are to create a spatial environment and an atmosphere for performance. Depend on the digital medium, the narrative content has gradually extended from time sequence to space distribution, reinterpreting the relationship of time and space in narration. Due to the improvements of carrying capacity of digital information and expression method in space scene, the montage once used in television and film are now being adopted on stage. Performance can split, select, and reorganize stage elements in a nonlinear narrative approach to attract audiences' attention and improve their immersive experience from multi-dimensions.

\section{Digital media design strategy for stage}

Four Orders of Design

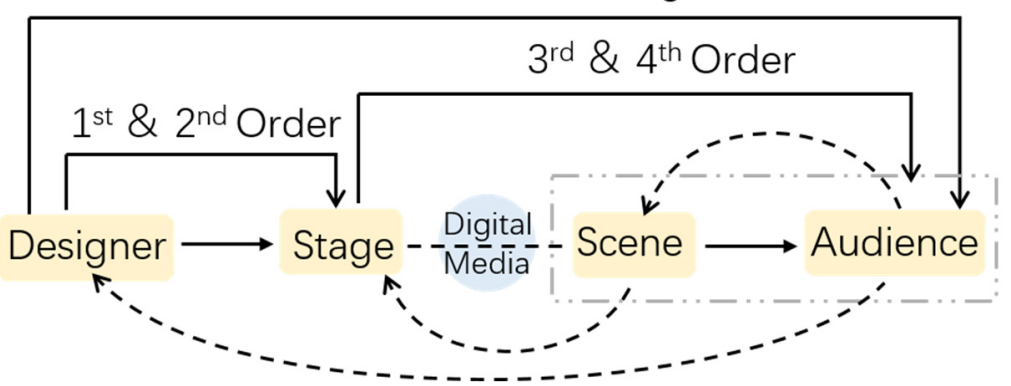

Figure 4. Four Orders of Design for stage

The process of stage design can be simply abstracted to connect audiences to stage performance through designers' efforts to create scene on stage to achieve best audiovisual experience. Due to the application of digital technologies on stage, the whole process of stage design turns to be more complicated. When designing the stage, designers should first consider how the scene will influence audiences' experience from the perspective of interactivity, experience and immersion, and then combine the application strategies of digital media technologies in different levels of needs to optimize the stage design. In this way, the design practice once belonging to the first and second order in the four orders of design has gradually extended to interactive design in the third design order and continuously to systematic design in the fourth order(figure 4).

Under the guidance of four orders of design, to apply digital media technology in stage design, a comprehensive and complete system is needed first and foremost. This system takes digital stage design server as the core, and at present various integrated server system like Disguise, Hecoos, SMODE can perform the function. To begin with, this digital stage design server must be able to support the import of the digital model of stage, build multi-channel timelines based on program scripts and produce and edit all types of digital image and video. Moreover, this server must also be able to accurately design and control digital media items like electronic screens, projections, mechanical devices, lighting consoles, MIDI consoles, virtual cameras, etc. Lastly, this server should also be open and scalable, and could control open source design system, tracking design system and interactive design system.

By establishing above-mentioned system framework, following the process of the four orders of design, the digital media technologies for stage design have been extended in five levels: content expression, interaction technology, spatial environment, psychological sensibility and communication effects, and finally meet the needs of safety, creativity, artistry, culture and empathy. 


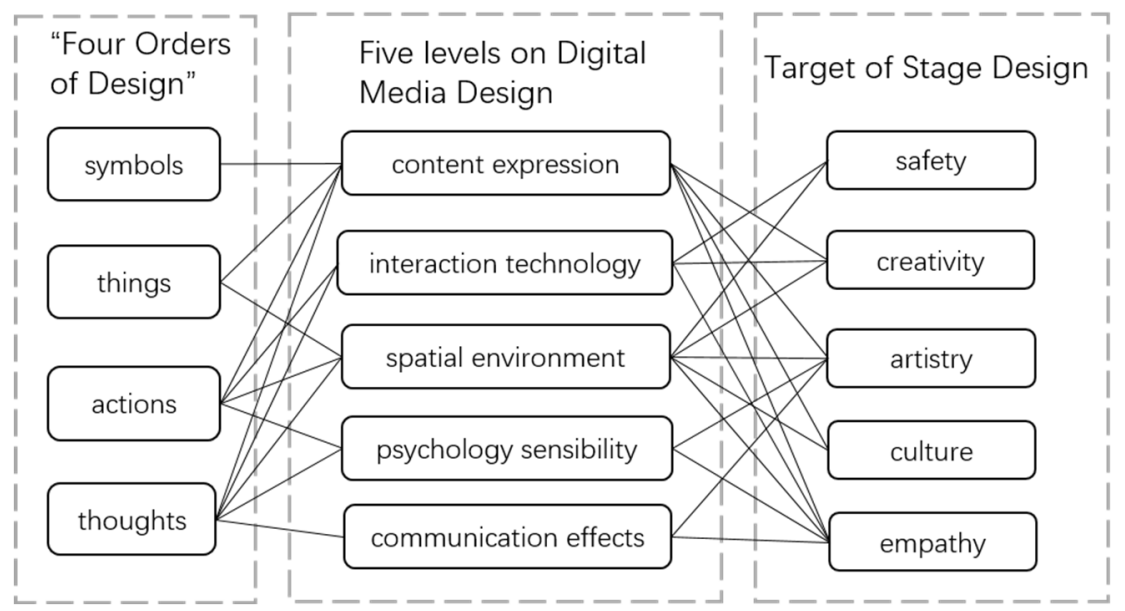

Figure 5. Five levels on digital media design between Four Orders of

Design and target of stage design

Safety: Besides ensuring the artistic performance, the responsibilities of stage designers also include consider the effective organization of overall space and guarantee the technical parameters of the stage safety especially on the level of interactive technology applications and spatial environment design. The safety of stage design will also benefit from the increasingly mature digital simulation systems and the technical support of digital media technology via different types of software and hardware in the links of information recording, storage, processing, transmission, display, management and so forth.

Creativity: Digital media design for stage transforms traditional stage design's creation concept and idea, making stage art a medium to pass information. From a practical perspective, it digs deep into the design innovation of digital media on the level of content expression, interactive design and spatial environment shaping thus presenting the richness and diversity of stage performance.

Artistry: A comprehensive art manifests when digital media technology deeply interconnects scene, lighting and sound on stage. This artistic temperament, which can penetrate into the content expression, spatial environment, psychological sensibility and communication effects of the stage, will become the most remarkable feature in stage design and inspiring the audiences' infinite imagination.

Culture: On stage, if safety is the foundation, creativity the trend and artistry the essence, then culture will be the soul. Culture is the connotation of content expression and spatial environment for digital media design of stage. With the crowding of various forms and styles into the stage art, the cultural appeal of stage design will upgrade aesthetic conception and lead audience to deeper thinking.

Empathy: Digital media technologies free stage design from single narration and enhance the interaction among environments, actors and audiences. Through integrating virtual image and spatial scenes in different ways, digital media technologies create a more immersive audiovisual experience. Moreover, by establishing effective connections between interaction technology and spatial environment, content expression and communication effects, it creates strong resonance and vivid audiovisual experience among audiences.

\section{Conclusion}

Stage art, as a comprehensive art, develops and evolves closely with drama, technology and aesthetics. As a contemporary extension of stage art, digital media for stage design presents new perspectives and ideas bridging the gap between art and technology, time and space, virtuality and reality, and provides a new design path for the development of stage art. Under the guidance of the four orders of design, stage design, with the help of digital media, will change from partial focus to overall consideration, develop from specialization to interdisciplinarity, transform from pre-production to realtime interaction, evolve from atmosphere creation to narrative expression, and will realize the safety, creativity, artistry, culture and empathy requirements of stage design.

\section{References}

1. Buchanan, R. (2001) Design thinking and the new learning. Design Issues, 17, 4: 3-23.

2. Buchanan, R. (1992) Wicked problems in design thinking. Design Issues, 8, 2: 5-21.

3. Han, S. (2008) Introduction to Stage Design. Culture and Art Publishing House, Beijing.

4. Gillette J M, Dionne R. (1987) Theatrical Design and Production: An Introduction to Scene Design and Construction, Lighting, Sound, Costume, and Makeup. Mayfield Publishing Company, Huston.

5. Brewster K, Shafer M. (2011) Fundamentals of Theatrical Design: A Guide to the Basics of Scenic, Costume, and Lighting Design. Skyhorse Publishing Inc., New York.

6. Aronson, A. (2012) The dematerialization of the stage. In: Aronson, A. (ed.) The Disappearing Stage: Reflections on the 2011 Prague Quadrennial. Arts and Theatre Institute, Prague.pp.86-95. 